\title{
De la promoción de la lectura por placer a la formación integral de lectores
}

\author{
Elsa Margarita Ramírez Leyva *
}

Artículo recibido:

23 de enero de 2014.

Artículo aceptado:

14 de mayo de 2015.

\section{Resumen}

En artículo tiene el objetivo de proponer elementos para renovar la formación de lectores ante la complejidad de la lectura que hoy exigen las sociedades del conocimiento. En este contexto surgen concepciones sobre la función de la biblioteca como espacio de formación, aprendizaje, cultura y construcción de sociabilidades; por ello la bibliotecología debe desarrollar una propuesta con una perspectiva de lectura más amplia que la de los modelos pedagógicos en las instituciones educativas. Se identifican en la Bildung aportaciones para una formación de lectores integral, se incorporan además una variedad de recursos que fa-

* Instituto de Investigaciones Bibliotecológicas y de la Información de la UNAM, México.eramirez@unam.mx

INVESTIGACIÓN BIBLIOTECOLÓGICA, Vol.30, Núm.69, mayo/agosto, 2016, México, ISSN: 0187-358X. pp. 95-120 
vorecen la lectura de diferentes códigos para ampliar el capital cultural y léxico que a la vez generan modalidades de lectura dirigidas al desarrollo de capacidades de pensamiento crítico y de reflexión involucradas en la construcción de conocimiento, así como para causar experiencias estéticas necesarias en la formación y transformación subjetiva de los ciudadanos a lo largo de su vida.

Palabras clave: Formación de Lectores y Bibliotecología; Lectura y Sociedades del Conocimiento; Bildung; Alfabetización Múltiple.

\section{Abstract}

Encouraging reading for pleasure and the comprehensive training for readers

Elsa Margarita Ramírez-Leyva

This paper proposes an approach to renewing the teaching of readers how to cope with the complex demands of the information society. In this context, the role of the library in training, learning, and construction of sociability and culture requires examination; and librarianship needs to develop broadly conceived proposals that move beyond pedagogical reading models offered schools. Bildung provides guidance for the comprehensive training of readers across a variety of codes, thereby equipping trainees with reading skills needed to develop broader lexicons and other associated tools and cultural capital that are useful in the construction of knowledge. Moreover, such training enriches the subject with aesthetic experiences that have the potential to effectuate subjective transformation of citizens over the long term.

Keywords: Reader's Education; Library Science; Reading and Knowledge Societies; Bildung; Multiple Literacies. 
Si lo que uno quiere es educarse y formarse, es de fuerzas bumanas de lo que se trata, y en que, sólo si lo conseguimos, sobreviviremos indemnes a la tecnología y al ser de la máquina.

Hans-Georg Gadamer

Time has turned library into school and the librarian is a teacher, at his best, and the visitor is a reader among books as a worker is among his tools.

Melvil Dewey

\section{INTRODUCCIÓN}

T as sociedades del siglo XXI necesitan lectores capaces de hacer uso de Lla información para transformarla en conocimiento. En este contexto la formación de lectores es una de las responsabilidades de los bibliotecólogos que se ha desplegado en dos vertientes: una, dirigida al incremento de la práctica de la lectura asidua y por placer, la cual se extiende cada vez más a grupos de adultos, inclusive a comunidades de educación superior; la otra, realizada en el ámbito de los servicios de usuarios en la modalidad de educación o formación de usuarios. Y ahora también se incorporan las habilidades informativas o alfabetización informativa ante los cambios en las modalidades de lectura, acceso, selección y el uso de la variedad de contenidos escritos, además de la gama de recursos audiovisuales y géneros hipertextuales que generan las innovaciones de los recursos electrónicos, lo cual implica cambios en los modos de leer, informarse e informar.

El escenario del presente siglo requiere innovar la función de formar lectores en el campo bibliotecológico, la cual se torna más compleja debido a las habilidades que ahora se exigen para transformar el recurso informativo en conocimiento, el cual se revaloriza como denominador de la sociedad actual y se considera un elemento estratégico para impulsar las economías, la innovación, la competitividad y la interacción global. La producción de información -cada vez más abundante y que se transmite de manera constante por medio de artefactos móviles en todo momento y en casi cualquier lugar del planeta- y su uso se hacen imprescindibles ya que tienden a cubrir la mayor parte de las actividades cotidianas, laborales, de aprendizaje, entretenimiento y comunicación. Por lo mismo, los ciudadanos deben desarrollar habilidades de lectura para potenciar las capacidades en un entorno global competitivo, por lo que hoy es fundamental resolver los factores de riesgo.

Entre los problemas que aún persisten y que para algunos países limitan su integración plena en las sociedades del conocimiento se encuentran las bre- 
chas sociales, una de ella propiciada por las carencias o deficiencias en los procesos educativos; el analfabetismo, el analfabetismo funcional de las comunidades que no han tenido oportunidad de ejercitar sus habilidades de escritura y lectura. A ello se agregan las deficiencias de lectura, identificadas en las evaluaciones internacionales y nacionales de diferentes países, entre alumnos que han concluido la educación básica, media superior y superior. Asimismo, se ha encontrado en la última década una disminución de la práctica de la lectura asidua por placer entre jóvenes en proceso educativo. Estos problemas afectan a las sociedades que buscan homogeneizar el dominio de la lectura y el aprendizaje de habilidades informativas y comunicativas en sus ciudadanos.

En suma, si bien la abundancia de información a la que ahora es posible acceder, las oportunidades que brindan las tecnologías para aprovechar recursos en educación, actividades laborales y productivas, la actualización permanente y el entretenimiento ofrecen oportunidades para el avance social, también pueden tornarse adversas en las comunidades que padecen discapacidades de lectura, de habilidades informativas y de comunicación. Lo anterior no se limita sólo a comunidades desfavorecidas, sino que también se extiende a comunidades de niveles superiores de educación, condiciones en las que pueden agudizarse las brechas cognitivas, culturales, sociales y económicas. De tal manera, el presente configura un contexto en el que la bibliotecología requiere renovar los modelos de formación de lectores y la educación de los bibliotecólogos para el desempeño de la práctica bibliotecaria, a fin de contribuir en las soluciones de los problemas que limitan las oportunidades para acceder y usar los contenidos que ofrecen los diferentes recursos, no sólo destinados a fines utilitarios y cotidianos, sino también para el desarrollo pleno de las capacidades de cada persona: cognitivas, reflexivas, críticas, dialógicas, creativas, imaginativas, afectivas, contemplativas y lúdicas, de asombro, curiosidad, del libre albedrío y de gozo intelectual y estético. Todas ellas están involucradas en la formación a lo largo de su vida no sólo con fines utilitarios sino también para formar ciudadanos que participen en cambios sociales para el beneficio propio, de sus comunidades y del ecosistema, y en el desarrollo de un mejor entorno. Por lo tanto, es necesario transformar y fortalecer la biblioteca como un espacio alternativo de apropiación de la información, de aprendizaje, de formación, de esparcimiento, de construcción de sociabilidades que ya no se limitan al ámbito local, pues ahora se extienden a diferentes puntos del planeta y abren nuevas modalidades multiculturales de convivencia como parte de la globalización.

La responsabilidad formadora de la bibliotecología no significa una función educativa en el sentido de la pedagogía que se practica en la escuela, sino que debe crear alternativas más allá del aprendizaje escolar, de los ámbitos laborales, familiares y del interés del consumo comercial. Por ello, pro- 
ponemos que la función bibliotecológica de la formación de lectores potencie la lectura y la información en un amplio sentido, en cuanto a variedad de códigos, prácticas, usos, que además de resolver necesidades escolares, académicas, laborales y cotidianas estimulen las capacidades de los individuos necesarias en su formación y transformación, lo que implica apropiarse, empoderarse y responsabilizarse del lenguaje y de la información, lo cual no se alcanza de una vez y para siempre, sino que se requiere de un desarrollo constante y permanente. Así, cada individuo tiene opciones para convertirse en actor y no en objeto de la palabra escrita y de los poderes discursivos que nos rodean y que ahora se extienden en el ámbito digital.

Desde diferentes perspectivas se enuncia la función de la formación de lectores como parte de la responsabilidad social de la biblioteca, en especial en el panorama de las sociedades del presente siglo, en el que se están transformado las maneras leer, informarse e informar. Por tal motivo, el objetivo de este artículo es analizar las propuestas sobre la función formadora de la biblioteca e identificar elementos para renovar el discurso y los modelos de la educación bibliotecológica y de la práctica bibliotecaria sobre la formación de lectores, que ahora deben incorporar contenidos en diferentes códigos, ampliar los fines más allá de la promoción de la lectura placentera e incluir otras capacidades que contribuyan al desarrollo de la alfabetización académica, las habilidades informativas o la alfabetización informativa, necesarias para los ciudadanos de las sociedades del conocimiento.

\section{LA LECTURA Y LA INFORMACIÓN, ¿AGENTES DE RIESGO EN LAS SOCIEDADES DEL SIGLO XXI?}

El siglo XXI se orienta a desarrollar las sociedades del conocimiento, pero también sabemos que en cada época las comunidades han concebido sus propias maneras de generar información, comunicarla y transformarla en conocimientos; gracias a ello se han logrado procesos civilizatorios, pero no se han resuelto problemas de rezagos, exclusiones o brechas sociales. Ahora, la peculiaridad de los conocimientos científico-tecnológicos, como afirma León Olivé, es que se desplazan hacia un lugar central, en tanto que el recurso del que depende la producción e insumos en los sistemas de innovación, cuyos resultados consisten en productos, procesos, formas de organización, sistemas o servicios, son aplicados en la solución de problemas y en la obtención de beneficios para algún grupo humano. ${ }^{1}$ Agrega que el énfasis 
en una auténtica sociedad del conocimiento debe estar en la educación y las condiciones que garanticen el desarrollo de las capacidades para aprovechar el conocimiento ya existente, para generar el nuevo que se requiera para la solución de problemas y para la realización de los planes de vida, así como en que exista una efectiva disponibilidad pública del acervo universal de conocimiento. $^{2}$

En efecto, no es suficiente la vasta información a la que es posible acceder ni la más innovadora tecnología, pues paradójicamente éstas pueden convertirse en factores de riesgo en las comunidades que enfrentan discapacidades en la lectoescritura y en las habilidades informativas y comunicativas, de las que no están exentos quienes han alcanzado la educación superior. En estas condiciones, ya se advierten rasgos de preocupación en los señalamientos expresados por la propia Unesco y la Federación Internacional de Asociaciones de Bibliotecarios (IFLA), así como en los diferentes autores que identifican elementos problemáticos que harían más profundas las brechas cognitivas, sociales y generacionales entre países y al interior de las comunidades y que de alguna manera coinciden en lo siguiente:

- La brecha cognitiva se abre entre quienes tienen acceso a una mejor educación, infraestructura, recursos informativos de calidad y habilidades para seleccionarlos, validarlos, utilizarlos y aprovecharlos para generar e innovar conocimiento, y por tanto tienen mejores oportunidades de desarrollo personal y laboral. ${ }^{3}$

- El uso excesivo de tecnologías electrónicas en edades tempranas puede tener efectos adversos en el proceso de desarrollo neurológico de los lenguajes oral, escrito y visual, en la maduración motriz y afectiva. De igual manera, los modelos pedagógicos que privilegian la rapidez en las etapas iniciales del aprendizaje de la lectura pueden tener efectos negativos en el nivel neuronal al exigir capacidades que no todos los organismos alcanzan en el mismo tiempo, pues maduran a ritmos diferentes y de manera progresiva con lectura oral, en silencio, deletreo y escritura manual. ${ }^{4}$

- Centrarse en la pantalla de manera excesiva sustrae los sentidos y la percepción que se requieren para leer los signos y extraer la información sobre la realidad que nos rodea. El uso de tecnología sin límites

2 Ibid., 24

3 Unesco, Hacia las Sociedades del Conocimiento; véase también IFLA (International Federation of Library Associations and Institutions), ¿Surcando las olas o atrapados en la marea? Navegando el entorno en la evolución de la información.

4 Stanislas Dehaene, El cerebro lector: Últimas noticias de las neurociencias sobre la lectura, la enseñanza, el aprendizaje y la dislexia, 235 ss. 
puede propiciar una creciente dependencia a ella, lo que provocaría el debilitamiento de las capacidades humanas que siguen siendo esenciales, pues por más perfeccionadas que sean las máquinas hasta ahora, no han sustituido a las personas en el proceso de transformar la información en conocimiento, trabajo en el que leer es parte imprescindible. ${ }^{5}$

- La excesiva cantidad de información y su constante innovación, las capacidades humanas rebasadas para discernir sobre su calidad y la tendencia de pasar de una lectura a otras favorecen lecturas fragmentadas, rápidas, superficiales, por lo que se dificulta la reflexión y su asimilación.

- Los medios masivos y el Internet ofrecen entretenimiento y una extensa gama de contenidos, muchos de ellos banales, que podrían conducir hacia un tipo de sociedad dirigida a una diversión generalizada. La rapidez, variedad y facilidad para acceder a la información y a medios de entretenimiento permiten la comunicación en todo momento y en cualquier lugar por los artefactos móviles, lo que puede conformar una masa acomodada en su ignorancia, fascinada por la tecnología y cada vez más alienada e indiferente, sin asumir su responsabilidad ciudadana, a diferencia de otra formada por los expertos en los saberes productivos; así, con una masa poco preparada será económicamente insostenible este modelo social. ${ }^{6}$

- Las diferentes culturas pueden estar en riesgo al desvanecerse las tradiciones y conocimientos teóricos y prácticos que las sustentan, ya que se le da especial importancia a la innovación, sin considerar que las sociedades del conocimiento requieren de la memoria y transmisión del saber. ${ }^{7}$

- La superespecialización propicia la fragmentación del conocimiento, pues procura en poco la construcción de lazos con otros saberes, provocando una estrechez en la visión del todo, ya que se sabe mucho de una parte sin tener conciencia de lo que se ignora; esto puede generar "certezas" que provocan problemas éticos, de comunicación y de incomprensión, en donde se privilegian las lecturas de la especialidad y se reducen u omiten lecturas que pueden ampliar el horizonte, por ejemplo, la literatura o los ensayos de diferentes temáticas. ${ }^{8}$

5 M. Serres, Los cinco sentidos. Ciencia, poesía y filosofía del cuerpo, 23.

6 Antoni Brey, Daniel Innerarity y Gonçal Mayos, La sociedad de la ignorancia. Una reflexión sobre la relación del individuo con el conocimiento en el mundo hiperconectado, 38.

$7 \quad$ Ibid., 51 ss.

8 Edgard Morin, Los siete saberes necesarios para la educación del futuro, 19-23. 
- Excluir de la formación las variadas posibilidades de las artes plásticas, la fotografía, el cine, el teatro o la música, las cuales pueden ayudar al individuo a hacerse de un patrimonio de cultura general, a conocer y pensar las problemáticas humanas y a contextualizar la información de la especialidad, de tal modo que se reduce la posibilidad de establecer relaciones e influencias recíprocas entre las partes y el todo, en un mundo cada vez más complejo, dado que ahora se producen interrelaciones multiculturales con efectos sociales en una dimensión mundial. ${ }^{9}$

- Las bibliotecas pueden enfrentar conflictos para cumplir su misión de ofrecer acceso gratuito a los recursos informativos y de lectura ante el modelo de privatización del conocimiento, que puede extenderse al libro en su formato tradicional y en todos los que hacen posibles las TIC, por el dominio de la obtención de ganancias de las empresas que los producen, muy al contrario de la difusión del conocimiento como bien público cuya producción y accesibilidad debería considerarse como parte de la infraestructura que requiere cualquier sociedad contemporánea, de cuya existencia y operación, entonces, no deberían desentenderse los Estados ni los organismos internacionales. ${ }^{10}$

A todo lo anterior se suma la declinación de la lectura asidua por placer. De acuerdo con las evaluaciones de lectura realizadas por el Programa para la Evaluación Internacional de Alumnos (PISA, Programme for Internacional Students Assesment), la lectura por gusto disminuyó cinco puntos entre los años 2000 a 2009; además, se afirma que una diferencia crucial entre los estudiantes que tienen un buen rendimiento en la evaluación de lectura y los que tienen un mal rendimiento reside en que lean diariamente por placer, en lugar de cuánto tiempo dediquen a leer. Dicha institución asevera que en promedio los alumnos que leen diariamente por placer tienen una puntuación superior a un año y medio de escolarización a los que no lo hacen. ${ }^{11}$ En México, por ejemplo, la Encuesta Nacional de Lectura de $2012^{12}$ mostró que no han variado los índices desde el 2006. En Estados Unidos se encontró que la lectura por gusto ha disminuido en los últimos veinte años, especialmente entre jóvenes entre los 18 y 24 años de edad; también se señala que existe una relación entre la lectura cotidiana por placer con el logro académico, ya que

9 Idem.

10 Olivé, op. cit., 28.

11 PISA, OCDE, “¿Leen actualmente los estudiantes por placer?”.

12 Fundación Mexicana para el Fomento de la Lectura, A. C., "De la penumbra a la obscuridad. Encuesta Nacional de Lectura 2012. Primer informe”. 
mejora la comprensión de la lectura, el estilo de escritura, el vocabulario, la ortografía y la gramática. ${ }^{13}$

Frente a este panorama surgen consideraciones sobre la necesidad de renovar la función de la biblioteca, que tradicionalmente es parte del sistema social de comunicación que preserva y facilita la articulación entre la información registrada y las diferentes comunidades; por lo mismo, también tiende a ser revalorizada como parte de las soluciones de los problemas de lectura y de acceso y uso de la información. La formación de lectores se encuentra entre las contribuciones que nuestra disciplina puede aportar a la construcción de las sociedades del presente siglo, lo cual obliga a innovar el paradigma de la biblioteca como un diverso espacio de formación.

\section{LA BIBLIOTECA: ESPACIO DE APRENDIZAJE, FORMACIÓN, CULTURA Y SOCIALIZACIÓN}

La formación de lectores como parte de las funciones de la biblioteca del siglo XXI se encuentra plasmada en propuestas que provienen de diferentes perspectivas teóricas que la conciben como espacio de aprendizaje, formación, socialización y desarrollo de las habilidades informativas. La biblioteca se considera una alternativa para reducir diferentes brechas cognitivas, sociales, culturales y generacionales, de manera que su participación es parte de las alternativas para reducir o evitar los riesgos de dirigirnos a una sociedad desinformada, inculta e ignorante.

La Unesco, en su informe Hacia las sociedades del conocimiento, reafirma que en el siglo XXI es necesario desarrollar pensamientos críticos y que las posibilidades de Internet o la oferta multimedia no deben excluir los instrumentos auténticos del conocimiento como la prensa, la radio, la televisión y la escuela. ${ }^{14}$ Asimismo concibe el aprendizaje como un proceso fundamental para equilibrar a las sociedades en cuanto a brechas sociales, culturales, cognitivas y digitales, por lo que en esta perspectiva se considera que "las bibliotecas se pueden convertir en protagonistas fundamentales del desarrollo, favoreciendo la reducción de la extremada polarización de nuestro mundo en el acceso a los bienes culturales y la información". ${ }^{15}$ También destaca el hecho de que en tanto es un espacio de aprendizaje, "la biblioteca -desde el bibliobús hasta el gran complejo arquitectónico contemporáneo- seguirá

13 Heidi Gauder, Joan Giglierano y Christine H. Schramm, "Encouraging recreational reading among college students", 3 .

14 Unesco, op. cit., 18.

15 Ibid., 71. 
siendo un pilar de la circulación social de los conocimientos y un factor de vitalidad para las redes de aprendizaje. En efecto, sus funciones cognitivas y evolutivas hacen de ella una organización de aprendizaje por excelencia”. ${ }^{16}$

La idea de la biblioteca como espacio de aprendizaje no es nueva. Si nos remontamos a las sociedades del antiguo Egipto y Pérgamo, encontramos que sus bibliotecas eran espacios de aprendizaje e investigación; de igual manera las bibliotecas monásticas se constituyeron en un espacio de formación. El surgimiento de la biblioteca pública hacia el siglo XIX respondió a la necesidad de ofrecer a los ciudadanos alfabetizados la posibilidad de continuar su autoaprendizaje; tiempo después, en los años sesenta del pasado siglo, la biblioteca se renueva y adquiere significado como parte de la concepción de las sociedades de aprendizaje, que empiezan a modificar el paradigma de aprendizaje, al considerarse que no sólo debía circunscribirse al ámbito escolar ni a un proceso en tiempos determinados y definitivos, sino que debía prolongarse y dar mayor importancia a la actualización permanente, pues los individuos necesitan seguir aprendiendo para realizar diferentes actividades. Después, en el Informe Faure de la Unesco de 1972, se afirmaba que "la educación ha dejado de ser el privilegio de una elite y de estar vinculada a una determinada edad; tiende a ser coextensiva a la vez con la totalidad de la comunidad y con la duración de la existencia del individuo". ${ }^{17}$ Entonces se destaca que es más importante "aprender a aprender, reflexionar, dudar, adaptarse con la mayor rapidez posible y saber cuestionar el legado cultural propio respetando los consensos. Estos son los pilares en los que deben descansar las sociedades del conocimiento". 18

Jorge Larrosa propone que es responsabilidad del profesor mantener la biblioteca como espacio de formación, cuestión que pocas veces suele ocurrir en las instituciones educativas de nuestro país. Por lo mismo, es el bibliotecólogo el que debe asumir una doble responsabilidad: hacia el interior de la biblioteca en el sentido de formación que propone el autor y renovar los lazos sociales con las comunidades mediante proyectos innovadores y de apoyo a sus actividades y necesidades. La concepción de la lectura como formación que propone Larrosa se orienta en el sentido de Bildung, es decir, que va más allá de los fines escolares. En la medida en que los individuos aprenden y asumen la responsabilidad de su formación, construyen su propia identidad, configuran su particular humanidad y se convierten en lo que son. ${ }^{19}$ 
Para Larrosa la experiencia tiene muchas posibilidades críticas y prácticas en el campo educativo. En cuanto a las experiencias de lectura, producen algo en el individuo, por tanto la lectura no es un proceso que se agota al aprender la lección, sino en entender el contenido y prolongarse hacia un infinito que abre el texto -o cualquier otro medio que sea susceptible de lectura-; ese infinito que convoca al lector a seguir fuera del texto, en su proceso de formación, en tanto logre una experiencia de lectura. Al respecto, Larrosa afirma que la experiencia es cada vez más difícil por el exceso de información; el énfasis contemporáneo en constituirnos como sujetos informantes e informados no hace otra cosa que cancelar nuestras posibilidades de experiencia. Agrega que el sujeto de la información sabe muchas cosas, se pasa el tiempo buscando información, cada vez sabe más, pero en esa obsesión por la información y por el saber (pero por el saber no en el sentido de "sabiduría" sino en el sentido de "estar informado") lo que consigue es que nada le pase, y puede correr el riesgo de excluir las experiencias de lectura que requieren de tiempo y que se relacionan con la sensibilidad, el tacto y la piel, la voz y el oído, la mirada, el sabor y el olor, el placer y el sufrimiento, la caricia y la herida, la mortalidad. ${ }^{20}$ Estas experiencias de lectura abren la conciencia, el pensamiento crítico, la reflexión la imaginación, la creatividad. Este enfoque aporta elementos para considerar las peculiaridades de la experiencia en la formación de lectura que tiene beneficios en el ámbito educativo.

Por lo que respecta a la biblioteca como espacio para crear sociabilidades, Roger Chartier considera que uno de los propósitos de "las bibliotecas del mañana podría ser reconstituir alrededor del libro las sociabilidades que hemos perdido". ${ }^{21}$ Su propuesta se deriva de sus pesquisas sobre la historia de las trayectorias de la cultura escrita, en las que ha encontrado diferentes maneras en que la lectura ha construido vínculos sociales. Por ello, su propuesta se orienta a reivindicar la lectura como posibilidad de comunicación, de diálogo y de momentos compartidos alrededor de lo escrito. Agregaríamos del electrónico, porque ahora en el espacio virtual la lectura social es ya una realidad, porque con los libros que incluyen distintas aplicaciones se hace posible compartir, dialogar, intercambiar ideas y experiencias y plantear dudas. De igual manera, las modalidades del blog, los booktubers o las redes sociales, en donde se comparten lecturas y se intercambian comentarios, sugerencias, etc., forman parte de las sociabilidades en torno al texto. Es interesante analizar la propuesta de Chartier ante las bibliotecas tradicionalistas que han naturalizado, en sus espacios destinados a la lectura, el modelo pe-

20 Ibid., 105-106, 111.

21 Roger Chartier, "Muerte o transfiguración del lector", 114-115. 
dagógico de la lectura como estudio en solitario y silencio, lo cual deja poco margen a la socialización. Por ello, señala el autor que se "deben multiplicar las ocasiones y las formas para que los lectores tomen la palabra alrededor del patrimonio escrito y de la creación intelectual y estética. De ese modo, pueden construir un espacio público fundado sobre la apropiación crítica de lo escrito". 22 Agrega que "En un futuro que es ya nuestro presente, estos efectos serán los que, colectivamente, sepamos construir. Para bien o para mal, esa es hoy nuestra responsabilidad común". 23

Encontramos otro aspecto de la biblioteca como espacio para construir sociabilidades en la Declaración de Túnez sobre las bibliotecas, la lectura y el diálogo intergeneracional, suscrita por la Sección de Alfabetización y Lectura de la IFLA. En dicha declaración se expresa el papel de las bibliotecas como espacios en donde la lectura sea un medio de cohesión social y de diálogo intergeneracional, solidaridad y de experiencias; las bibliotecas también contribuyen a reducir las brechas de las comunidades en la medida en que la lectura y la información favorecen la inclusión social al integrarse en la formación de lectores. En el documento se reitera que el aprendizaje es una actividad a lo largo de la vida, que se facilita por la lectura y el acceso a la información. ${ }^{24}$

Desde la perspectiva bibliotecológica, Álvarez Zapata y otros colegas proponen que la función educativa de la biblioteca pública "se debe a los lectores en una doble perspectiva: la de servir como institución de la lectura y como espacio de expresión, y la de promover la ciudadanía". 25 Asimismo, resultan reveladoras las concepciones de los bibliotecarios que participaron en el estudio sobre la relación de la biblioteca pública con la lectura y los lectores, como señalan los autores: "la persistencia de una notable (y vieja, por lo demás...) dicotomía entre biblioteca y aula. Unas veces para separarse de ella en términos de una nueva práctica formativa de lectores, que propone que la promoción de la lectura impulsada por la biblioteca debe ser diferente (por renovadora o por placentera...) a la promoción de la lectura impulsada por la escuela". ${ }^{26}$ En cuanto a la otra perspectiva, ve a la biblioteca como una "institución en sí misma educadora con la tarea de apoyar la educación permanente y con una perspectiva de educación social" ${ }^{27}$ Consideran que si bien

22 Ibid., 115.

23 Ibid., 116.

24 iÚnete a IFLA. Sección de Alfabetización y Lectura!

25 Didier Álvarez Zapata, Yicel Nayrobis Giraldo Giraldo, Norfi Yamili Ocampo Molina, Luz Marina Guerra Sierra, Liliana Melgar Estrada y Maricela Gómez Vargas, "Representaciones bibliotecarias sobre la biblioteca pública, la lectura, el lector, la promoción y la animación a la lectura en Medellín, Colombia”, 235. 
el discurso bibliotecario sobre su relación con la cultura escrita tiene aún un camino que seguir en su consolidación científica, "sí encierra un rico conjunto de valoraciones y posibilidades comprensivas sobre el mundo social que, normalmente, se pierde o se enmascara detrás del discurso técnico y administrativo". ${ }^{28}$ Sobre esta consideración agregaríamos que la bibliotecología más tradicional concibe el acto de leer a la luz de la educación conservadora y del discurso editorial que enfatiza la lectura, en especial, de libros y por placer.

Otra de las propuestas sobre la relación de la biblioteca, los lectores y la lectura se encuentra en el discurso inaugural que José Ortega y Gasset leyó en el Segundo Congreso Internacional de Bibliotecarios de la IFLA, en Madrid, el 20 de mayo de 1935 y al que tituló Misión del bibliotecario. En ese escrito se esboza una filosofía bibliotecológica que remite a un aspecto ontológico, ya que enfatiza el "ser" bibliotecario y el "hacer" con estas palabras: "lo que cada hombre tiene que hacer para ser lo que es y la misión profesional". ${ }^{29}$ Y agrega: "al ejercer una profesión, se compromete a hacer lo que la sociedad necesita". $\mathrm{Al}$ respecto, en una de sus sentencias encontramos estas valiosas palabras: "Ahora se siente la necesidad, no de buscar libros -esto ha dejado de ser verdadero problema-, sino la de fomentar la lectura, la de buscar lectores. Y, en efecto, en esta etapa las bibliotecas se multiplican y con ellas el bibliotecario"." "Tendrá el bibliotecario del porvenir que dirigir al lector no especializado por la selva de los libros y ser el médico, el higienista de sus lecturas". ${ }^{2}$ A Ortega y Gasset le preocupaba que los lectores combatirían contra la "selva" de los libros y entonces el bibliotecario tendría que guiarlos para no perderse entre ellos, lo que hoy es también válido en este inmenso mar de información que crece constantemente, de ahí que en la disciplina bibliotecológica han surgido los programas de desarrollo de habilidades informativas.

La bibliotecología ha renovado sus modelos de educación o formación de usuarios al incorporar el desarrollo de las habilidades informativas o alfabetización informativa, que se orientan a la búsqueda, la recuperación y el uso de la información en diferentes lenguajes, especialidades y formatos; en algunos de esos modelos se incluye la modalidad de la lectura crítica. ${ }^{33}$ Este tipo de lectura es indispensable para discriminar, elegir y utilizar información ante su abundancia y variedad.

28 Idem.

29 José Ortega y Gasset, Misión del bibliotecario, 40.

30 Ibid., 41.

31 Ibid., 53.

32 Ibid., 78.

33 irinazey, "October is National Information Literacy Awareness Month". 
Las propuestas descritas en torno a la biblioteca como espacio de aprendizaje, formación y socialización en el contexto del siglo XXI son propicias para renovar el discurso y la práctica bibliotecológica sobre la formación de lectores, en la que se articulen elementos para ser, hacer y pensar ante los riesgos y ventajas que ofrece el universo de información, pero, sin duda, exige el desarrollo de capacidades en las cuales la lectura es factor primordial en el desarrollo de las sociedades.

\section{La CONTRibución de La BILDUNG PARA LA} FORMACIÓN DE LECTORES EN EL ÁMBITO BIBLIOTECOLÓGICO

La bibliotecología tiene la responsabilidad social de innovar sus contribuciones ante el desarrollo de las sociedades del siglo XXI, las cuales ponderan la información como factor de transformación. Este panorama es propicio para renovar el paradigma respecto de la función educadora de la biblioteca, ya no en el sentido tradicionalista de complemento de la escuela según se desprende de los discursos antes descritos, sino que se deben ampliar los alcances de intervención y trascender a un desafío mayor: una formación integral de lectores en la que se amplíe y fortalezca la lectura no sólo de los códigos escritos, sino extenderlos a los medios audiovisuales para el aprovechamiento de distintas fuentes informativas. Resulta indispensable, entonces, incorporar las alfabetizaciones de diferentes tipos, como la digital, la visual, la sonora, la matemática, la geométrica espacial, la social, la histórica, la cultural, la cenestésica, incluso la táctil, la olfativa y la gustativa. Estas variaciones renuevan las modalidades de lectura y de información que habían quedado subvaloradas pero que realizamos de manera cotidiana sin considerar que implican una decodificación y que son fuentes de información y experiencias, como bien nos recuerda Roland Barthes:

Cuando voy por la calle —o por la vida — y encuentro estos objetos, les aplico a todos, sin darme cuenta, una misma actividad, que es la de cierta lectura: el hombre moderno, el hombre de las ciudades, pasa su tiempo leyendo. Lee, ante todo y sobre todo, imágenes, gestos, comportamientos: este automóvil me comunica el status social de su propietario, esta indumentaria me dice con exactitud la dosis de conformismo o de excentricidad de su portador, este aperitivo (whisky, pernod, o vino blanco) el estilo de vida de mi anfitrión. Aun cuando se trata de un texto escrito, siempre nos es dado leer un segundo mensaje entre las líneas del primero. ${ }^{34}$ 
La bibliotecología, ante la responsabilidad de renovar la biblioteca como espacio de formación, aprendizaje y socialización, tiene en el enfoque de la Bildung de la pedagogía alemana los elementos para constituir un discurso propio respecto de la formación de lectores, ya que apunta a que los sujetos aprendan a utilizar diferentes recursos para el desarrollo de sus capacidades, a fin de formarse de manera integral, responsable y ética a lo largo de la vida. Este enfoque considera cuatro dimensiones centrales, las cuales C. E. Noguera retoma de Klafki:

1. Capacitación para la autodeterminación racional, en donde la autoactividad es la forma central de realización del proceso formativo, expresado mediante los conceptos de autodeterminación, libertad, emancipación, autonomía, razón y autoactividad.

2. La condición de la formación es la existencia de una cultura humana anterior, por tanto se privilegian las producciones humanas, las ganancias civilizatorias en la satisfacción de las necesidades, el conocimiento sobre la naturaleza y el ser humano, las acciones políticas, los sistemas de normas y acciones éticas, las formas de vida social y los productos estéticos.

3. Implica una relación dialéctica entre la individualidad y la colectividad, el diálogo es necesario para el proceso de formación.

4. La actividad humana, la cual debe ser el objetivo de la Bildung, consiste en tres actividades:

- Moral, que es la responsabilidad moral autodeterminada.

- Cognitiva, que se orienta a una vinculación con la reflexión racional sobre el sentido humano y la responsabilidad de sus posibilidades de aplicación, que debe estar presente como una pregunta permanente en relación a las posibilidades y límites de la racionalidad instrumental para una existencia humana del hombre.

- Estética, que se refiere al perfeccionamiento de la facultad sensitiva frente a los fenómenos de la naturaleza y de la expresión humana. Dicha sensibilidad requiere del desarrollo de la facultad imaginativa o fantasía, del gusto y de la facultad de gozo y juicio estéticos, así como de la capacidad para el juego y para la sociabilidad no sólo en el sentido de la formación de las personas en la literatura, el teatro, la música o las artes plásticas, sino también en la idea de una estética de lo cotidiano.

Estas dimensiones pueden concebirse, afirma Noguera, como la formación general de los seres humanos, y las refiere a una triple formación: la ca- 
beza, el corazón y la mano; podríamos agregar una más: el interés múltiple. Asimismo, señala el autor que en la Bildung el desenvolvimiento del sujeto en el mundo objetivo universal se logra cuando éste alcanza la racionalidad como parte de un proceso de apropiación y discusión crítica de la cultura, lo cual no se reduce a un plan de estudios, sino que es una formación no conclusiva que abarca la totalidad de la vida. ${ }^{35}$

Es importante resaltar la concepción que tiene E. Rodríguez Moncada sobre el término "formación" o Bildung: "supone el reconocimiento de los propios saberes, los adquiridos durante las experiencias de vida y durante la práctica; mientras dichos saberes y conocimientos no se reconozcan, los sujetos no se valorarán y seguirán colocándose en el lugar de la ignorancia o serán colocados ahí por los otros". ${ }^{36}$ Destaca que esta formación está dentro y fuera de los espacios de educación,

no por ello deja de formarse el individuo: cuando se lee un libro, cuando se conversa con compañeros, cuando se disfruta una melodía o una película, el sujeto entra en relación con objetos (textos distintos aprehensivos de la realidad), con los cuales vive una experiencia, aprende y, por tanto, se forma. En este sentido cabe también reconocer la importancia de la llamada educación informal. ${ }^{37}$

Por otro lado, la Bildung -afirman M. Deimann y R. Farrow- conduce a una cultura participativa porque el aprendizaje no es sólo cognitivo, sino también social y emocional, no obstante que dichos elementos han estado exiliados de las teorías que se sustentan en modelos tradicionales de aprendizaje. ${ }^{38}$ Posiblemente esto se deba a que la Bildung puede resultar un empoderamiento de las personas, pues como dice V. Gómez Ibáñez: "en tanto que apropiación subjetiva de la cultura, implica la posibilidad de "esclarecimiento" (Erbellung) de la conciencia individual, su ilustración progresiva en orden a la ilustración del todo, de la sociedad: cuanto más esclarecido el individuo, tanto más esclarecido el todo social". ${ }^{39}$

Jorge Larrosa, como ya se mencionó, incorpora en sus concepciones sobre la lectura como formación elementos de la Bildung, ya que la relaciona con la subjetividad del lector, es decir, no sólo con lo que sabe sino con lo es: no es sólo una forma de entretenimiento o una lectura para el aprendizaje,

35 Carlos Ernesto Noguera Ramírez, Aproximación conceptual a la constitución de las tradiciones pedagógicas modernas, 4-6.

36 Ernesto Rodríguez Moncada, "Reflexiones en torno a la formación y la práctica de educadores de adultos", 140

37 Ibid., 140-141.

38 Markus Deimann y Robert Farrow, "Rethinking OER and their use: Open Education as Bildung".

39 Vicente Gómez Ibáñez, "La liquidación de la filosofía. Notas sobre la disputa entre R. Rorty y J. Habermas", 124. 
que es para lo que forma la escuela. Larrosa destaca en la lectura como formación la importancia de la experiencia, y nos recuerda que la experiencia de la formación no era otra cosa que apropiarse de lo que estaba recogido en esas palabras memorables que se guardaban en la biblioteca; la experiencia de la lectura es un modo de relación con el texto en la que esa apropiación queda asegurada. ${ }^{40}$ Pero, agrega, ahora esa experiencia de lectura poco se practica en el ámbito escolar y no favorece que el lector se apropie de los contenidos, ya sea por los tiempos rápidos y los resultados determinados en los programas que se exigen en el espacio escolar o por la cada vez más voluminosa cantidad de información accesible en las variadas modalidades de comunicación y esparcimiento en el medio digital que lo mantienen entretenido, lo cual dificulta asimilar la información porque ahora "una experiencia [...] [es] algo [que] se llena y se vacía indefinidamente" por la velocidad y la cantidad de información. ${ }^{41}$ Para la Bildung la experiencia es uno de los elementos fundamentales de la formación, incluso las neurociencias le reconocen su importancia como un elemento involucrado en los procesos de renovación neuronal, células en las que se plasman huellas hechas de informaciones que tienen efectos en varios procesos involucrados en la producción de conocimiento y en las creaciones artísticas. ${ }^{42}$

La Bildung se ha empezado a reconsiderar como una alternativa frente a los problemas actuales de la educación identificados por PISA en diferentes países en aspectos de lectura, matemáticas y ciencias, a lo largo de un poco más de diez años. Se han revisitado los modelos educativos con expertos en este campo, entre ellos Kotthoff y Pereyra, quienes reconsideran a la Bildung "para orientar los cambios educativos que introducen las nuevas tecnologías de las comunicaciones y la cultura virtual, [que son] significativos para un tiempo en el que la identidad, la subjetividad, la filosofía del sujeto y lo que debe significar un nuevo humanismo, deberían problematizarse con más atención y cuidado". ${ }^{43}$ Los autores citados identificaron los aspectos esenciales de la Bildung después de que la filósofa Christina Schües los actualizara, de ellos destacamos los siguientes:

- Toda persona es libre. Schües considera que en esta formación, si bien los conocimientos son importantes, no lo son todo, pues de la misma manera se debe fomentar la aspiración, el amor y la motivación perso-

40 Larrosa, "La experiencia de la lectura", 568.

41 Ibid., 594.

42 François Ansermet y Pierre Magistretti, "Neurociencias y psicoanálisis".

43 Hans-Georg Kotthoff y Miguel A. Pereyra, "La experiencia del PISA en Alemania: recepción, reformas recientes y reflexiones sobre un sistema educativo en cambio", 21. 
nal (que pueden aplicarse al conocimiento, aunque no sean necesarios para éste).

- El campo específico de ejecución de la Bildung abarca desde el concepto de humanidad en cuanto a las relaciones de tensión hasta la formación del mundo. Esto implica ser audaz y significa ampliar los horizontes de la información y la orientación del pensamiento (ambos implican la capacidad para comparar y reflexionar sobre diferentes tipos de modos de pensar).

- Establecimiento de la duda entre la unidad de racionalidad y entre la pluralidad de mundos, historias y culturas. Schües apoya dicha antinomia, diciendo que es una autorreflexión que no está subordinada al pensamiento estratégico, sino que demuestra una meditación (un alto consciente) que conduce al discernimiento, y a poseer la habilidad para llevar un estilo de vida propio y responsable, lo cual implica la obtención de poder. ${ }^{44}$

En la bibliotecología, autores como E. Naranjo o J. Verdugo han revisado la noción de "formación" del concepto alemán de Bildung ${ }^{45}$ e identifican elementos en los que son indispensables la lectura, la experiencia, la información y la comunicación. El discurso bibliotecológico y la práctica bibliotecaria sobre la formación de lectores puede encontrar elementos para orientar la filosofía del ser y del quehacer de sus profesionales en los postulados de la Bildung, pues tenemos la responsabilidad de renovar el paradigma bibliotecario para fortalecer la función de la biblioteca como un espacio de lectura e información para el aprendizaje, la formación y la socialización, así como el acceso y uso de la cultura, con la intervención de los bibliotecólogos, para lograr que las personas y el registro gráfico se encuentren en una fructífera experiencia intelectual. ${ }^{46}$ Así, cada persona podrá desarrollar las capacidades cognitivas, reflexivas, críticas, dialógicas, creativas, imaginativas, afectivas, contemplativas y lúdicas, de asombro, de curiosidad, de libre albedrío y de gozo intelectual y estético, estimuladas, que no suplantadas, por la lectura y la información. Todas ellas participan en la formación y transformación de la subjetividad, de los saberes y de las experiencias, ya que el ser y el saber no se producen de una vez y para siempre, sino que se labran a lo largo de la vida, no sin dificultades y esfuerzo para el logro de la plenitud.

45 Edilma Naranjo Vélez, "Formación de usuarios de la información y procesos formativos: hacia una conceptuación”, 40 ss; José Alfredo Verdugo Sánchez, "Hacia un concepto de formación de usuarios y propuesta de un programa", 4-6.

46 Jesse Shera, Fundamentos de la educación bibliotecológica, 41. 


\section{LA ALFABETIZACIÓN MÚLTIPLE EN LA FORMACIÓN INTEGRAL DE LECTORES}

La función social de la biblioteca puede potenciarse en las sociedades del conocimiento que exigen de los ciudadanos un mayor dominio de la lectura para elevar el rendimiento académico, laboral y el aprendizaje a lo largo de la vida. Ante ello, la bibliotecología puede contribuir con propuestas que expandan la promoción de la lectura hacia otras posibilidades, como la lectura crítica, la académica, el diálogo entre la literatura, las artes y las ciencias, la construcción de sociabilidades, la alfabetización múltiple y el desarrollo de habilidades informativas.

Los programas de formación de lectura pueden incorporar una gama amplia de recursos que favorezcan el placer, el deseo de leer y las distintas modalidades de lectura. Los recursos que pueden integrarse a las actividades lúdicas de lectura ofrecen al mismo tiempo una variedad de temas de diferentes campos de conocimiento para la lectura crítica o la comparativa, la investigación, conocer diferentes culturas (dado que en algunos países se promueve la formación multicultural). Además de apoyar temas especializados se enriquece el vocabulario y se amplía la cultura, se favorece el desarrollo de los diferentes sentidos, de las experiencias sensibles y de los gozos intelectuales. Con todo ello, en la biblioteca se pueden ampliar y diversificar los usos de la lectura, a la vez fortalecer las habilidades que implican las lecturas más profundas, y reconstruir las representaciones negativas que alejaron o frustraron los encuentros gratos con la lectura en la trayectoria de los lectores.

La alfabetización múltiple es considerada por D. Masny como una variedad de textualidades con múltiples significados que se encuentran en los espectros visual, oral, escrito, táctil, olfativo, digital, multimodal. Constituyen textos, en un sentido amplio, la música, las artes visuales (pintura, escultura), la física, las matemáticas, las combinaciones digitales que se fusionan con la religión, el sexo, la raza, la cultura y el poder. Estas alfabetizaciones se actualizan de acuerdo con un contexto particular en el tiempo y en el espacio en el que operan. Masny observó un enriquecimiento de las capacidades de pensamiento y asociaciones más complejas en comunidades que leen diferentes códigos. ${ }^{47}$

A los géneros académicos y de la literatura escrita se pueden incorporar la lectura de los variados códigos que ofrecen las artes plásticas, el cine, la ópera, el teatro, la danza, los cómics, las noticias y los programas de los medios masivos, radio y televisión, los géneros electrónicos también ofrecen distintos códigos como la modalidad de los hipertextos, blogs y videojuegos, sin 
olvidar los recursos que ofrece la tecnología electrónica para construir sociabilidades mediante la lectura y la información, como los fanfics, las redes sociales, los booktubers, la lectura social. Otras formas de lectura singulares que también amplían los espectros de la lectura son los fenómenos naturales, la arquitectura, los elementos del mundo de la naturaleza, los deportes.

Todos los recursos antes enumerados implican modalidades de lectura específica, para lo cual es necesario aprender a leer los códigos en los que están expresados. Esto lleva a una alfabetización múltiple que amplía el aspecto informativo y la lectura crítica, comparada, profunda, a fin de desentrañar más allá de lo aparente, incluso puede favorecer una lectura hermenéutica, semiótica, en donde también se encuentran elementos simbólicos que se utilizan por ejemplo en el cine o en las artes plásticas, y que son propicios para la investigación. Esas formas de leer pueden motivar la reflexión, el deseo de saber y el placer, o pueden generar experiencias sensibles. La variedad de códigos activa distintas zonas del cerebro relacionadas con la memoria, la asociación de informaciones, de experiencias, de sensaciones, de alegría, enojo, tristeza o gozo. Así, entre mayor variedad y novedad de información reciba el cerebro de los sentidos, la activación neuronal favorecerá la expansión de las capacidades humanas que ya hemos señalado, de las que depende la producción de conocimientos, la invención y las artes.

La lectura de diferentes códigos también contribuye al desarrollo de las habilidades involucradas en las alfabetizaciones que son parte del aprendizaje de los lenguajes de las diferentes disciplinas que se impulsan en la alfabetización académica e informativa. La bibliotecología ha desarrollado modelos más versátiles, por ejemplo el propuesto por el National Forum on Information Literacy de la American Library Association (ALA), que entre sus categorías incluye la alfabetización multimedia (visual y computacional) y la alfabetización crítica (lectura crítica y el pensamiento crítico). ${ }^{48}$ El modelo Iceberg $^{49}$ que propone S. Kurbanoglu abarca habilidades y alfabetizaciones de diferentes códigos escritos y audiovisuales.$^{50}$ En el modelo de Dianne McKenzie se incluyen diferentes alfabetizaciones: la comunicacional, que abarca lectura, escritura, expresión oral, escuchar; la visual, la histórico-cultural, la multimedia, la digital, la matemática, la científica, la de redes sociales, la financiera, la musical, la gráfica y la cenestésica. ${ }^{51}$

48 irinazey, op. cit.

49 El modelo Iceberg indaga sobre los elementos que no son perceptibles y es necesario profundizar para conocerlos y comprender los factores que producen los resultados visibles que son la punta del iceberg, lo que permite una mayor comprensión del mundo y utilizarlos para que se produzcan o se modifiquen, ejemplo de ello pueden ser los modelos mentales.

50 Serap Kurbanoglu, "An Analysis of Concept of Information Literacy", 83.

51 Dianne McKenzie, "Information literacy is the basis for all learning". 
Los distintos recursos escritos y toda la gama de medios visuales y sonoros se han legitimado en el siglo XXI como fuentes de información y como objetos de lectura y de cultura. Recordemos que U. Eco y R. Barthes hablaban desde el siglo pasado de los diversos signos que se encuentran en el mundo social, cultural y natural que vinculan los canales sensoriales con el modo como las personas reciben determinados signos clasificados por la mente. Así, el destinatario recibe señales procedentes de los canales sensoriales y los transforma en mensajes. ${ }^{52}$ Para ambos autores, de la lectura de esos signos que nos rodean se puede extraer tanto información para la construcción de conocimiento como capital cultural para la maduración de las capacidades involucradas en la percepción. Todo esto esclarece la información que los ciudadanos leen en los elementos, hechos, objetos o mensajes que conforman los contextos y que participan en su formación.

Hoy en día las habilidades de lectura se ha tornado más complejas, por ello nuestra propuesta es ampliar los programas bibliotecarias más allá de la búsqueda del placer per se para promover el deseo de saber, de indagar, de reflexionar, de imaginar, de crear, de conocer más allá de lo evidente, es decir, una lectura más diversa de la que los individuos se apropien para desarrollar un patrimonio informativo, cognoscitivo y cultural más robusto y versátil del que pueden obtener los elementos para lograr una formación integral, tal como lo propone la Bildung. Así, en la medida en que se forja una ilustración subjetiva progresiva, se va logrando la ilustración del todo y de la sociedad en sí: cuanto más esclarecido sea el individuo, tanto más esclarecido será el todo social y la posibilidad del Erhellung.

La biblioteca puede ser, entonces, ese espacio en donde se entrecruzan los saberes de los productos humanos de todas las épocas, favorables para el aprendizaje, la formación, la cultura, la información; propicio para el esclarecimiento, el descubrimiento y el gozo. Las bibliotecas tendrán que considerar la diversificación de sus colecciones y generar bibliografías amplias y diversas para el apoyo de programas escolares y académicos, además de los culturales. Pero también el bibliotecólogo tendrá que cultivar una cultura amplia y variada. En este sentido, J. Shera consideraba que el conocimiento fundamental del bibliotecólogo debe ser de una amplia cultura; además, afirmaba que dicho conocimiento sólo puede entenderse desde el punto de vista de la responsabilidad social que desempeña la biblioteca. Independientemente del tipo de usuarios "el interés del bibliotecólogo radica en la interacción de mentes humanas comunicándose a través de las barreras de espacio y tiempo, valiéndose de los registros gráficos, cuyo contenido puede entregarse 
por medio de los sentidos, del sonido y del tacto, así como de la vista, pues se entiende que un registro gráfico puede ser audible y táctil, así como visual". 53

En esta idea el autor alude a una concepción más amplia de los recursos informativos; cabe señalar que desde hace décadas las bibliotecas más modernas inspiraron medios audiovisuales, al contrario de las bibliotecas tradicionalistas que se habían enfocado en el desarrollo de colecciones impresas como fuentes de información legítimas; sin embargo, en la actualidad la tendencia es diversificar los acervos. Por otro lado, la cultura digital que también es escrita ha contribuido a renovar su interacción con los códigos audiovisuales que conforman géneros hipertextuales y que también ofrecen una gama muy amplia de recursos para diferentes actividades científicas, académicas, educativas, laborales, productivas, lúdicas, estéticas y domésticas, así como de posibilidades de comunicación por medio de códigos escritos y audiovisuales.

\section{ConClusión}

En el marco del proyecto de sociedades del conocimiento, resulta oportuno recordar dos señalamientos de Jesse Shera: "la base del sistema bibliotecario es la comunicación de información, en donde ésta debe entenderse como cualquier manifestación gráfica de una actividad intelectual. Así, el bibliotecólogo debe ver al mundo profesionalmente como un intrincado modelo de comunicaciones." En relación con ello, Shera afirma que la "aptitud del bibliotecólogo dependerá de su habilidad para abstraer un sistema a partir del mundo intelectual, emocional, social y físico que lo rodea -el mundo que él 'sirve". 54

Se necesita un modelo bibliotecológico para las sociedades del siglo XXI, que se caracterizan por cambios profundos en el modelo cultural que privilegia el conocimiento en un contexto social global que exige producción e innovación, articulado por la tecnología electrónica que acelera la producción e intercambio de información, por no dejar de lado las comunicaciones. En este contexto se transforman los modos en que los ciudadanos aprenden, se informan, crean, conocen, se comunican, socializan, se entretienen y usan la información, la cual crece de manera exponencial a una mayor velocidad y rapidez de la que aquéllos la transmiten desde cualquier parte del mundo. Todo ello nos abre posibilidades -como nunca antes se habían tenido- para 
generar, comunicar, acceder y usar contenidos, lo que ha dado lugar a asegurar que entre más informados y formados estemos llegaremos a progresar. Sin embargo, esas ventajas de sobreabundancia, rapidez y posibilidades de disponer de los contenidos de forma constante pueden producir riesgos debido a las limitaciones humanas para leer, analizar, reflexionar, asimilar saberes y tener experiencias, sobre lo cual miradas críticas ya alertan de la posibilidad de convertirnos en sociedades de la ignorancia, el desconocimiento y la incultura, al pensar de manera cómoda que los medios, la industria informativa y la tecnología reducirán y hasta eximirán los esfuerzos y tiempo que debemos invertir en informarnos y formarnos.

Ante este panorama la biblioteca puede constituirse en un espacio donde la ciudadanía puede optar por una trayectoria distinta a los determinismos culturales, sociales y educativos al apropiarse de la lectura y la información para forjarse como los ciudadanos responsables de la construcción de las sociedades del conocimiento. La institución bibliotecaria cobraría una mayor dimensión en el sostenimiento de su formación o Bildung, que sería integrada por la cultura escrita, las artes, el diálogo, la interacción social, las experiencias, las habilidades personales y sociales. Pero la biblioteca debe hacer que la lectura se despliegue en una diversidad de posibilidades bajo el dominio de lectores cada vez más expertos; así, los sujetos se formarían en la medida en que potencian sus capacidades y se hacen responsables de su desarrollo y con ello, de su destino.

\section{BiBLIOGRAFÍA}

Álvarez Zapata, Didier, Yicel Nayrobis Giraldo Giraldo, Norfi Yamili Ocampo Molina, Luz Marina Guerra Sierra, Liliana Melgar Estrada y Maricela Gómez Vargas. "Representaciones bibliotecarias sobre la biblioteca pública, la lectura, el lector, la promoción y la animación a la lectura en Medellín, Colombia”. Investigación Bibliotecológica 23 (49) (2009): 197-240.

Ansermet, François y Pierre Magistretti. "Neurociencias y psicoanálisis". Cuadernos de psiquiatría y psicoterapia del niño y del adolescente 43/44 (2007): 5-16.

Barthes, Roland. Lo obvio y lo obtuso. Imágenes, gestos y voces. Barcelona: Paidós Ibérica, 1986.

"La cocina del sentido", en La aventura semiológica, 2a. ed. Buenos Aires: Paidós Comunicación, 1993, 223-225.

Brey, Antoni, Daniel Innerarity y Gonçal Mayos. La sociedad de la ignorancia. Una reflexión sobre la relación del individuo con el conocimiento en el mundo hiperconectado. Barcelona: Libros Infanzonía, 2009. 
Chartier, Roger. "Muerte o transfiguración del lector", en Las revoluciones de la cultura escrita. Diálogos e intervenciones. Barcelona: Gedisa, 2000, 101-119.

Dehaene, Stanislas. El cerebro lector: Últimas noticias de las neurociencias sobre la lectura, la enseñanza, el aprendizaje y la dislexia. Buenos Aires: Siglo Veintiuno Editores, 2014.

Deimann, Markus y Robert Farrow. "Rethinking OER and their use: Open Education as Bildung". The International Review of Research in Open and Distance Learning 14 (3) (2013). Disponible en: http://www.irrodl.org/index.php/irrodl/article/view/1370/2542 [Fecha de consulta: 10 de noviembre de 2013].

Eco, Umberto. Signo. Barcelona; Labor, 1994. Tratado de semiótica general, 5a ed. España: Lumen, 2000.

Fundación Mexicana para el Fomento de la Lectura, A. C. "De la penumbra a la obscuridad. Encuesta Nacional de Lectura 2012. Primer informe". México, 2013. Disponible en: http://www.educacion yculturaaz.com/wp-content/uploads/2013/04/ENL_2012.pdf [Fecha de consulta: 10 de febrero de 2013].

Gauder, Heidi, Joan Giglierano y Christine H. Schramm. "Encouraging recreational reading among college students". College $E$ Un dergraduate Libraries 14 (2) (2007): 1-24.

Gómez Ibáñez, Vicente. "La liquidación de la filosofía. Notas sobre la disputa entre R. Rorty y J. Habermas”. CONVIVIUM. Revista de filosofía 6 (1994): 124. Disponible en: www.raco.cat/index.php/ Convivium/article/download/73317/98529 [Fecha de consulta: 10 de noviembre de 2013].

IFLA (International Federation of Library Associations and Institutions). ¿Surcando las olas o atrapados en la marea? Navegando el entorno en la evolución de la información, 2013. Disponible en: http:// trends.ifla.org/files/trends/assets/surcando_las_olas_o_atrapados_en_la_marea.pdf [Fecha de consulta: 20 de febrero de 2014].

irinazey, "October is National Information Literacy Awareness Month", en THLNewsBlog, 9 de octubre de 2012. Disponible en: http:// thlibrary.wordpress.com/2012/10/09/october-is-national-informa tion-literacy-awareness-month [Fecha de consulta: 15 de junio de 2013].

Kotthoff, Hans-Georg y Miguel A. Pereyra. "La experiencia del PISA en Alemania: recepción, reformas recientes y reflexiones sobre un sistema educativo en cambio". Profesorado, Revista de currículum y formación de profesorado 13 (2) (2009): 1-24.

Kurbanoglu, Serap. "An Analysis of Concept of Information Literacy". Media and information literacy for knowledge societies. Moscú: Interregional Library Cooperation Centre, 2013: 1-42. Disponible en: http://www.ifapcom.ru/files/News/Images/2013/mil_eng_web. pdf [Fecha de consulta: 7 de noviembre de 2013]. 
Larrosa, Jorge. "La experiencia de la lectura”, en Estudios sobre literatura y formación, 2003. México: Fondo de Cultura Económica.

"Sobre la experiencia". Aloma: revista de psicologia, ciències de l'educació i de l'esport 19 (2006): 87-112.

Mckenzie, Dianne. "Information literacy is the basis for all learning". Library Grits, 1 de mayo de 2010. Disponible en: http://librarygrits. blogspot.mx/2010/05/information-literacy-is-basis-for-all.html [Fecha de consulta: 15 de junio de 2012].

Masny, Diana. "Multiple Literacies Theory: how it functions, what it produces". Perspectiva 28 (2) (2010). Disponible en: http://www. perspectiva.ufsc.br/ [Fecha de consulta: 23 de julio de 2012].

Morin, Edgar. Los siete saberes necesarios para la educación del futuro, 2a. reimp. Buenos Aires: Nueva Visión, 2002.

Naranjo Vélez, Edilma. "Formación de usuarios de la información y procesos formativos: hacia una conceptualización”. Investigación Bibliotecológica: archivonomía, bibliotecología e información 19 (38) (2005): 33-60. México.

Noguera Ramírez, Carlos Ernesto. Aproximación conceptual a la constitución de las tradiciones pedagógicas modernas, 2013. Disponible en: http://hdl.handle.net/11195/304 [Fecha de consulta: 20 de septiembre de 2013].

Olivé, León. "El libro, la lectura y las bibliotecas en la sociedad del conocimiento". Lectura y vida 30 (3) (2009): 20-29.

Ortega y Gasset, José. Misión del bibliotecario, ed. conmemorativa del 50 aniversario luctuoso del autor y de la celebración del Día Nacional del Bibliotecario. México: CONACULTA, DGB, 2005.

PISA, OCDE. “¿Leen actualmente los estudiantes por placer?”. PISA in Focus 8 (2011). Disponible en: www.oecd.org/pisa/pisaproducts/ pisainfocus/49184736.pdf [Fecha de consulta: 20 de septiembre de 2013].

Rodríguez Moncada, Ernesto. "Reflexiones en torno a la formación y la práctica de educadores de adultos". Revista Interamericana de Educación de Adultos 31 (1) (2009): 137-150. Disponible en: http:// www.crefal.edu.mx/rieda/images/rieda-2009-1/contrapunto1.pdf [Fecha de consulta: 10 de noviembre de 2013].

Serres, M. Los cinco sentidos. Ciencia, poesía y filosofía del cuerpo. México: Taurus, 2002.

Shera, Jesse. Fundamentos de la educación bibliotecológica. México: Universidad Nacional Autónoma de México (UNAM), Centro Universitario de Investigaciones Bibliotecológicas (CUIB), 1990.

Unesco. Hacia las Sociedades del Conocimiento. París: UNESCO, 2005.

Disponible en: http://unesdoc.unesco.org/images/0014/001419/ 141908s.PDF [Fecha de consulta: 19 de marzo de 2014].

¡Únete a IFLA. Sección de Alfabetización y Lectura! (s. a.). Disponible en: http://www.ifla.org/files/assets/literacy-and-reading/publications/ Tunis-declaration-spanish.doc [Fecha de consulta: 12 de agosto de 2012]. 
Verdugo Sánchez, José Alfredo. "Hacia un concepto de formación de usuarios y propuesta de un programa”. Investigación Bibliotecológica: archivonomía, bibliotecología e información 7 (15) (1993): 4-15.

Para citar este artículo:

Ramírez Leyva, Elsa Margarita. 2016. "De la promoción de la lectura por placer a la formación integral de lectores." Investigación Bibliotecológica: Archivonomía, Bibliotecología e Información 69: 95120. http://dx.doi.org/10.1016/j.ibbai.2016.04.014 